\title{
Volume 21 keyword index
}

1,3-butadiene 5643

$13 \mathrm{q} 216567$

14-3-3 $\sigma$ 2418, 3207, 8310, 1876

2-acetylaminofluorene 1945

2-deoxyribonolactone 8926

2-methyoxyestradiol 4149

2'-methoxyethyl RNA, telomere 631

$3 p 12$ tumour suppressor gene 3020

3'-phosphoglycolate 8926

4-disulphide core 2768

5-aza-2'-deoxycytidine 5496

5-azacytidine 5483

5-lipoxygenase 5765

7,8-dihydro-8-oxoguanine (8-oxoG) 8232

8-hydroxyguanine 1649

8-oxoguanine 8905

8oxo G/A mismatch 5204

A2B adenosine receptor 4663

A3 adenosine receptor 4060

A431 2670

av $\beta 6$ intergrin 1370

Abl 6848

ABL 8536

Acanthoma 6446

Acetyl-CoA carboxylase 6729

Acetylation 1556, 2741

Acetylcholinesterase 8428

Actin 96

Actin polymerization 5967

Actin rearrangement 1477

Activator protein-1 738

Active or open nucleosomes 1649

Activin 2227

Acute lymphoblastic leukemia 3225

Acute promyelocytic leukemia 3496

adCMV-vpr 4613
Adenine nucleotide translocator 1963

Adenocarcinoma 158, 5804, 5814, 7831

Adenoma 5814

Adenoma-carcinoma sequence 4120

Adenomatous polyps 7175

Adenovirus 87, 1411, 4757, 6278, 8011, 8340

Adenovirus gene therapy 3688

Adenovirus vector 2108, 2493

Adherens junction(s) 7067, 7642

Adhesion 8272, 8605, 8683

Adhesion molecule 3089

Adipose tissue 1679

Adrenomedullin 2815

Afadin 2545, 4108

Ageing 3872

Aging 494, 4363

Agnoprotein 5574

AIBI 7147

AIDS 3592, 7464

AIF 65

Akt 53, 207, 329, 337 , 1062, 1159, 1299, 1727, 1945, 1955, 3532, 4032, 5224, 7131, 7797, 8683, 8871

AKT 1391, 6255

AKT1 198

ALCL 1038

Alcohol 1539

Alcohol intake 1225

Alcoholic liver disease 32

Alkylating agent(s) 878, 1108,5940

All-trans retinoic acid 7147

Allele-specific 5753

Allelic imbalance 5975 , 8878

Allelic loss(es) 5016, 7605

Allelotyping 1461

ALT 522

ALT-associated PML bodies 598
Alternative first exons 6007

Alternative lengthening of telomeres 598

Alternative splicing 2768, 3029

Alternatively-spliced p53 5117

AML 4247

AML1 2695, 3232, 3247, 8428

AML1/ETO 3422

AMLI/Evi-1 2695

AMP-activated protein kinase (AMPK) 6082

Amplification 909, 2261, 7593

A-MuLV 8568

A-Myb 1859

Anaplastic lymphoma kinase (ALK) 5823

Anchorage 1777

Anchorage independence 3969

Anchorage independent growth 217,2365

Androgen receptor 2679, 5069, 5609, 8453

Androgen regulated genes 8749

Androgen-regulated 4738

Aneuploidy 6146

Angiogenesis 272, 427, 2058, 2245, 2433, 2504, 2815, 3172, 3541, 3889, $4765,5951,6307,8128$, 8506

Angiogenic factor 8477

Angiostatin 272

Animal model(s) 2089, 3445,9033

Ankryin 5255

Annexin I 6641

Anoikis 2161, 2365, 2908, 6801, 7797, 8001

Anoxia 4212

Ansamycins 1159

Antagonistic pleiotrophy 503

Anti-apoptotic genes 4166

Anti-cancer therapy 8786

Anti-estrogens 4000
Anti-neoplastics 9

Anti-neovascular therapy 2662

Antiangiogenesis 4558

Antibod(ies) 1304, 5725, 7114

Anticancer activity 3162

Anticancer drugs 4317, 4885

Antiestrogen 7147

Antiestrogen-resistance 8178

Antiestrogens 8158

Antigen 3879, 8668

Antisense 87, 2890, 3162, 8011

Antisense oligodeoxynucleotides 4166

Antisense RNA 5127

Antisense technology 4932

AP endonuclease 8905, 8926

AP1 738

AP-1 2181, 3121, 3836 , 4266, 5923, 6317, 6434, 7680,8186

AP-1 complex 2971

AP-2 7478

Apaf-1 1469, 1848

Apc 6446

APC 4646, 4696, 6387, 8340

APC promoter hypermethylation 7981

Ape1 protein 8926

Aphidicolin 5929, 7226

Apigenin 3727

Aplidin 7533

Apo2L 5763

Apo2L/TRAIL 3611

Apoptosis 1, 9, 32, 44, $65,140,190,227,260$, $319,329,337,377,532$, 592, 656, 748, 778, 878, 947, 1017, 1028, 1038, $1071,1135,1171,1233$ $1251,1285,1299,1335$, $1359,1381,1469,1563$, $1600,1668,1685,1727$, $1750,1759,1848,1882$, 1963, 1989, 2020, 2141, 2171, 2245, 2283, 2295, 
$2413,2441,2484,2534$ 2613, 2623, 2775, 2829, 2890, 2919, 2981, 2991, $3011,3082,3112,3121$, 3139, 3149, 3181, 3213 , 3260, 3284, 3459, 3532, $3727,3765,3796,3836$, 3872 , 3917, 3925, 4042, 4149, 4323, 4335, 4392, 4473, 4481, 4539, 4558, 4613, 4691, 4702, 4715, 4757, 4801, 4944, 4957, 5038, 5047, 5160, 5268 5716, 5835, 5844, 5886, 5897, 5934, 5951, 6091, $6113,6123,6132,6278$, 6299, 6458, 6510, 6598, 6606, 6649, 6772, 7050, 7195, 7514, 7524, 7861, $7901,8020,8114,8149$, $8223,8240,8361,8379$, 8404, 8414, 8568, 8591, 8804,8843

APRT 2840

A-Raf 347

ARE 2191, 5301

ARF 2939, 5960

Aromatase 2854

Arsenic trioxide 3496

Arylhydrocarbon receptor 6059

Asbestos 1434, 7284, 7471

ASK 7786

Asparagine 5891

Astrocytoma 1325, 4050

Ataxia-telangiectasia 849 , 3864, 4363

ATF 7824

ATF-2 1000

ATF3 7488

ATL 2466

ATM 483, 532, 611, 849, 1090, 4191, 4363, 6377, 6633, 7776

ATRA 5224

Attachment 4765

Aurora 6175

Aurora-B 3103

Auto-antibody 5006

Auto-antigen 5006, 5946

Autocrine 108, 6540, 8817

Autocrine TGF $\beta 7514$

Autocrine transforming growth factor $\alpha 2785$

Autoimmunity 5593

Avihepadnavirus 6606

AXIN1 4863, 7981

AXIN2 4863, 7981

Axl 329
BAD 329

BADD45 5335

BAG-1 isoforms 7050

Bak 227

Barrett's cancer 3780

Barrett's esophagus 475

Barrett's oesophagus 6071

Barrier function 3765

Basal cell carcinoma 5529

Base excision 8905

Base excision repair 5204, 8675

BATF 8186

bax 990

bax 6458

Bax 32, 1963, 2020, 3139 , 3149, 4009, 4530, 4757, 6032, 7050

$\beta$-catenin 856,2679 , 4099, 4863, 5861, 5906, 7861, 7971,8293, 8340, 8414,8453

$\beta$-catenin activation 5548

$\beta$ catenin mutations 7981

B-cell lymphoma 8173

B cell markers 4908

B cell phenotype 4166

B cells 3855

B-cell neoplasia 7235

bcl-2 6458

$\mathrm{Bcl} 24530$

BCL2 7765

Bcl-2 32, 1548, 2020, 3898, 4549, 4613, 4944, 5047, 5313, 5897, 7050, 7611,8149

Bcl-2 cleavage 2141

Bcl-2 family 748, 2762

BCL6 gene 368

BCL-family 708

Bcl-x 1668

Bcl-xl 6510

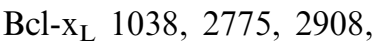
5182, 7765

$B C R / A B L 8536$

$B C R 8536$

Bcr 4601

BCR/ABL 5868, 8547, 8577, 8605

BCR-ABL 3068, 4351, 8612, 8629

Bcr-Abl 8075, 8804, 8541,8560

Bcr - Abl protein 8584

Bcr/Abl P190 3225

$\mathrm{BCR} / \mathrm{ABL}$ rearrangement 5716

Beta-catenin 6598, 6983
Betaig-h3 7471

bFGF 809

BH3 3181

Big Blue ${ }^{\text {TM }} 7126$

Bik 227

BIK 2534

Bile acids 6740

Bile duct cancer 4301

Bim 4944

Bioinformatics 475, 8029

Biological classification 8206

Biomarker(s) 6870, 7598

Bisindolylmaleimide-I 6348

Bisulphite genomic sequencing 1048

BK virus 5725

Bladder cancer 650, 2476, 4894

Bloom's syndrome 954, 2079, 2525, 9008

B-lymphoma 1922

BMP-2 7156

B-Myb 1859, 7923

Bone tumors 1141

B-Raf kinase 6356

Brain 3939

Brain tumor(s) 878, 1141, 2058, 5038, 8372

BRC repeat 1130

BRCA 6729

BRCA1 1401, 3199

Brcal 4747, 5097

BRCA1 4171, 6222,

7034, 7730, 8981, 8994

BRCA1 mutations 6841

BRCA1-associated surveillance complex 4788

BRCA2 1130, 5188, 5660, 8981, 8994

BRCT 6729

Break-induced replication 522

Breast cancer 108, 227, 460, 761, 836, 964, 1009, 1316, 1832, 2066, 2261, 2270, 2365, 2704, 2854, 3172, 3517, 3934, 4000, 4080, 4171, 4307, 4422, 4521, 4626, 4680, 4747, 4921, 5047, 5127, 5148, 5175, 5462, 5619, 5654, 5696, 6366, 6567, 7034, 7147, 7557, 7680, 7850, 8158, 8178, 8843

Breast cancer cell(s) 5765, 7730

Breast cancer model 890
Breast carcinoma(s) 3241, 4317

Breast neoplasms 8506

Breast tumor(s) 3532, 8529

Breast-ovarian cancer 6841

Brn-3a 6123

Bronchoscopy 6949

BTEB 2212

BTG2 $2^{\text {TIS21/PC3 }} 6772$

$\beta \operatorname{TrCp~856,~1501,~} 3978$

Burkitt cell line 4473

Butyrate 6340

C3H/10T1/2 8196

c-Abl 974

Cadherin 2545, 4108, 7642

Cadherins and catenins 6071

Calcium 2762

Calcium signaling 7839

Calponin 7720

cAMP 778, 4020, 4060

Camptothecin 7913, 8361

Cancer 494, 564, 598, 1309, 3089, 3917, 4435, 5380, 5394, 5400, 5414, 5483, 5496, 5951, 6071, 6082, 6140, 6154, 6175, 6234, 7307, 7656, 7749, $8741,8862,9033$

Cancer development 1679

Cancer diagnosis 475

Cancer predisposition 9008

Cancer prevention 6113

Cancer specific cytokine 708

Cancer-chemoprevention 3663

Cancer-related protein 4932

Cancers 688

Capping 532, 627

Carboplatin 2805

Carcinogenesis 2750, 3780, 4728, 5462, 6870, 8272,8895

Carcinogens 7435

Carcinoid 8310

Carcinoma cell 7514

Casein kinase II 5280

CASP8 8510

Caspase 377, 748, 809, 1251, 1299, 2020, 2534, 4613

Caspase-3 2108, 7050, 8047, 8414

Caspase-8 2295, 6132 
Caspase-9 2141

Caspase-10 2919

Caspase 104129

Caspase cleavage 867

Caspase-independent 6091

Caspases 65, 1017, 1242, 1848, 2283, 2623, 4089, 4530, 5160, 5313, 6032, 6132, 6649, 6809, 7533, 8843

Caspases, DN-caspase 9 1

Catalase 5886

Catenin 4108, 7042, 7656

Cathepsin B 8705

Cathepsin-D 5127, 5951

Cathepsin L 7839

Catnb 5643

Caveolin 1658, 2365

CBC 5649

CBF 3247

CBF $\beta 3422$

Cbl 1707, 5346

CBL-B 1423

CBP 2253, 2455, 7241

$\mathrm{CBP} / \mathrm{p} 3001028$

CCK-2R 7656

CCND1 1928

CD30 2493

CD31 4558

CD44 1196

CD9 2584

CD95 165, 1600, 4042, 6809

CD95/Fas 190

cdc2 6425

CDC25B 7630

Cdc25C 1717, 6633

CDC42 3939

Cdc6 1777

CDH1 8192

CDH1 1928

cdk 7765

CDK 8089

CDK4 2476

cdk inhibitors 4680 , 6573

cdk2 6573

Cdk2 6154, 8397, 8529

CDK4 4277, 8067, 8852

Cdk6 1777

CDK6 4277

cdk9 4137, 4158, 5797

Cdkn2a 5960

CDKN2A 8878

CDKs 6413

cDNA array 3095, 5804

cDNA arrays 4587, 4957

cDNA macroarray 2926 , 2270, 2634, 4120, 8521
CDNA microarrays 475 , 4855

CDT1 1150

Cdt1 6624

CEA 7817

C/EBP 3377

C. elegans 6201

Cell adhesion 1450, 3889, 7656, 8612

Cell attachment 8470

Cell cycle 140, 437, 447, 708, 1359, 1485, 1493, 1548, 1717, 1750, 1934, 2741, 2750, 3247, 3525, 3651, 3796, 4328, 4539, 4626, 4680, 4978, 5313, 5515, 5773, 5897, 6059, 6146, 6403, 6530, 6573, 6722, 7230, 7545, 7630, 7765, 7850, 7901, 7923, $8001,8067,8158,8282$, 8320,8804

Cell cycle arrest 5912, 7569

Cell cycle checkpoint(s) 2079, 4191, 6884

Cell cycle progression 5245

Cell cycle regulated protein 1123

Cell cycle regulation 6779

Cell death 809, 1963, 8262

Cell differentiation 2347 , 6772

Cell dispersion 1477

Cell growth 1658, 2029, 3784, 7891, 8498

Cell invasion 8240

Cell line 2114, 4646

Cell migration 1423, 8470

Cell morphology 6779, 6801

Cell motility 4089, 6520

Cell proliferation 2201, 3889, 5529

Cell scattering 2347

Cell shape 4843

Cell survival 207, 1062

Cell transformation 248, 4838, 7497

Cell viability 6017

Cell-cycle arrest 3727 , 6123

Cell-surface proteolysis 3765

Cell-cell adhesion 2719, 8414

Cell-cell junctions 1477 , 2545

Cell-cycle 1685
Cellular localization 6766

Cellular senescence 503

Central-nervous-system neoplasm 239

Centriole 6146, 6201

Centrosome 890, 5097, 6140, 6154, 6161, 6175, 6184, 6195

Centrosome amplification 6222, 6228

Centrosome duplication 6201, 6209

Centrosome hyperamplification 6234

Centrosomes 6241

Ceramide 44, 2020, 4009

Cervical cancer 165 , 5631, 6241, 8713

Cervix neoplasia 665

c-FLIP 6809

c-fos 2721

c-Fos 4843

c-fos repression 719

CGH 158, 1518

CGH analysis 6877

Chaperone activity 1123

Checkpoint(s) 1633, 2328, 6249, 8591

Chemoprevention 3727, 7945

Chemoresistance 7611, 8683

Chemosensitivity 1

Chemotherapy 260, 6059

CHFR 2328

Chk2 1316, 6633

Choline kinase 937, 4317

Chromatic structure 5358

Chromatin 719, 2741, 5361,6403

Chromatin remodeling 1196

Chromosomal abnormality(ies) 1679, 2525

Chromosomal instability $619,825,3253,6146$

Chromosomal rearrangements 7671

Chromosomal translocation 1309

Chromosome 12261

Chromosome 34521

Chromosome 41225

Chromosome 45135

Chromosome 4p 8356

Chromosome 6q21 7266

Chromosome 8p 1461

Chromosome 11p15.5 2564
Chromosome 11q13 5631

Chromosome 12q21 3029

Chromsome 136567

Chromosome 172097

Chromosome 185016

Chromosome 202768

Chromosome 22q 6480

Chromosome instability 4673, 5654, 6140, 6884

Chromosome segregation 7507

Chromosome translocation 6703

Chromosomes 2051

Chronic myelogenous leukemia (CML) 8560 , 8668

Cidofovir 2334

Cigarette smoke(ing) 7326, 7392, 7435

CIMP 2741

Circadian clock 9043

Circulating cancer cells 7817

Cisplatin 5935, 6713, $7131,8361,8723$

c-Jun 2441, 7077

c-Jun $\mathrm{N}$-terminal kinase 2154

c-Jun phosphorylation 2154

CK2 3978

CK20 7817

c-kit 3068

c-kit 4508

CLCP1 2822

Clb5 2051

Cleavage by caspase- 3 5024

Clinical application 6868

Clonality 2466, 3274

Clonogenic survival 5935

Cluster analysis 4855

Clusterin 4328

c-Maf 5301

c-Met 1000, 1791

c-Met tyrosine kinase 1477

CML 3068, 8541, 8547, $8577,8584,8605,8612$, $8629,8643,8804$

c-myb 3076

c-Myb 1859

c-myc 1600, 2097, 3414, 4384, 5280, 6848

c-Myc 909, 1518, 2981, 3011, 6559, 6819, 7872, 8414

c-Myc induction 7137

Co-activators 5773

Co-carcinogenesis 7392 
Co-transcriptional factor 861

Coactivator 7147

Cockayne syndrome 3571, 8675, 8949

Coding mononucleotide repeats 5081

Coiled-coil 4422

Collagen gels 5148

Collagenase 1187

Colon 8741

Colon cancer 1370, 1443 , 4120, 4646, 4855, 4992, 5861, 6032, 6387, 7256, $7797,7817,8340$

Colon carcinoma 1450 , 2785, 3688, 4997

Colorectal cancer 1928, 3253, 5441, 6740, 7175, $7277,7585,8477$

Colorectal carcinoma 5081, 5906, 6689

Combination therapy 5868

Common fragile sites 8713

Common virus integration site 7247

Companion animals tumours 1785

Comparative genomic hybridization 1117

Competitive PCR 1097

Complex translocation 4706

Conditional knockout 4635

Confocal microscopy 7710

Connexin 324992

Connexin 43 1989, 4992

Constitutive activation 78,5891

Constitutive phosphorylation 5823

Contact inhibition 5990

Conversion 6328

COX-2 7092

$\mathrm{CpG}$ island methylation 2114

CpG island(s) 1048, 3659, 3804, 5427, 5450

CpG methylation 1876, 8878

CPT-11 3611

CRE 5923

Cre 6446

c-Rel 8759

Cre-loxP system 3445

Cre-loxP 4521

Cre/lox 899
Crk1 8536

Crkl, Ph-positive 3225

Cross-antagonism 3368

Cross-talk 4000

Crypt 5441

Crystallin 1028

CSB 8232

c-Src 1071

CT element 7187

CtBP 2695

CtBP1 3232

Culture 7897

Curcumin 8414, 8852

Cyanide 282

Cyclin A 5791

Cyclin A2/Cdk2 7923

Cyclin B 2051

Cyclin B1 6751, 8282, 8696

Cyclin D 1159, 5791

Cyclin D1 198, 291, 307, 1590, 1928, 2212, 5515, $7545,8067,8852$

Cyclin D1 phosphorylation 7214

Cyclin D2 7214

Cyclin D3 1777

Cyclin $\mathrm{D}_{1}$ promoter 8001

Cyclin dependent kinase(s) 1750, 5031, 6908

Cyclin E 291, 4626

Cyclin E/Cdk2 7923

Cyclin E1 8529

Cyclin E2 8529

Cyclin T2 4158

Cyclin-cdk 8114

Cyclin-dependent kinase(s) 5773, 6779

Cyclin-dependent p21 kinases 3403

Cyclin(s) Cyclin 1263, 1493, 4137, 4680, 6154, 8089

Cyclo-oxygenase-2 411, 6113

Cyclobutane pyrimidine dimmer 5743

Cyclooxygeanse 7175

Cyclopentenone 2212

Cyclophilin 2261

Cyr61 964, 8178

Cystathionine beat synthase 6848

Cystatin C 8705

Cysteine 2191

Cyteine-proteases 8843

Cytochrome c 377, 1242, 1848, 2775, 3139, 4613, 5844

Cytochrome-P450 6059
Cytogenetics 6877

Cytokeratin expression 5548

Cytokine network 3284

Cytokine receptor(s) 3579, 6264, 7464

Cytokine(s) 2201, 3295, 4384, 8568, 8871

Cytology 643

Cytoplasm 1493

Cytoplasmic epoxide hydrolase 8521

Cytoskeleton 96, 1171, 3969,5255

Cytotoxicity 5213, 6264

$\Delta \mathrm{N}-\mathrm{p} 536722$

D-Loop 3780

DAP kinase 1217, 4231

Dbf4 7786

Dbl proteins 4601

DDA3 3050

Death and survival genes 4957

Death domain 4691

Death receptor 56032

Death receptors 319 , 2623, 3121, 6649

Decorin proteoglycan 3688, 4765

Degradation 2387

DEK 8862

Deletion 479, 1167, 4521

Deletion 7q 4849

Deletion in p53 4042

Deletion mapping 6915

Demethylation 789

Denys-Drash syndrome 2948

DEP1 7067

Dephosphorylation 1870

Desmoplastic small round cell tumor 2009

Detoxifying genes 2191

Development 2880

Dexamethasone 1346

DHBV 6606

dHPLC 5016

Diagnosis 643, 650, 5450, 6960

Diagnostic imaging 6949

Diethynitrosamine 1791

Differential expression 4932, 7598

Differentiation 128, 1527, 1600, 1859, 2880, 2971, $3068,3260,3262,3377$, 3403, 3414, 3739, 4289, 4747, 5268, 7933

Differentiation therapy 3496
Diffuse gastric cancer

8192

Diffuse large B-cell lymphoma 368

Dihydrofolate reductase 2981

Diphosphorylation 5852

DISC 6809, 8510

Disease gene 7247

Distinct gene profiles 2926

Dkcl deficient mice 7740

Dlg 8140

DMBT1 5956

DN-caspase 94530

DNA adducts 7376

DNA binding 981, 1563, 2119, 5117

DNA binding site 7901

DNA breakage 2141

DNA damage 356,553 , 592, 611, 974, 5844, 5912, 6614, 7435, 7488, $7776,8591,8723$

DNA damage repair 4481

DNA damage tolerance 8957

DNA double-strand break 6884

DNA double-strand break repair 4176

DNA glycosylase(s) 8886, 8905

DNA hypermethylation 6694

DNA hypomethylation 2564, 6694

DNA injection 3696

DNA instability 3571

DNA interstrand crosslink 5188

DNA methylation 1048, 3659, 3804, 3909, 4822, 5358, 5361, 5380, 5388, 5400, 5414, 5427, 5462, 5483, 5496, 9033

DNA methyltransferase 4822, 5361, 5483, 5496, 7883

DNA microarray(s) 437, 6848

DNA mismatch repair 1768, 5081, 5743, 7126

DNA photorepair 9043

DNA polymerase $\beta 2320$

DNA repair 437, 1401, 1633, 4065, 5188, 5204, 5335, 5394, 5654, 5940, 6471, 7901, 8232, 8591, 8886, 8935, 8981, 9022 
DNA replication 437, 909

DNA replication checkpoint 7710

DNA replication fidelity 8895

DNA synthesis 5929

DNA topoisomerase II 4706

DNA tumor virus 3207

DNA-PK 6471

DNA-binding 990

DNA-PK 532

DNA-repair 731, 3663

Dominance 1641

Dominant negative 2695 , 5990

Dominant negative mutants 2425

Dominant negative STAT3 4778

Double fusion 4181

Double minute chromosomes (DMs) 825,890

Double strand break 1401, 8967

Double strand break DNA repair 2525

Double-strand break repair 7671

Double translocation 4181

Down-regulated protein 6641

Downregulation 7131

Downstream target genes 8196

Doxorubicin 5160, 6132, 8020

Doxycycline 272

DR4 3121, 3611

DR5 3611

DRIP205 861

Drosophila 2413, 4812, 6458

Drug resistance 227 , $4595,8020,8843$

Drug targets 8786

Drugs 2295

DSRCT 1890

dsRNA 8379

DT40 954

DT40 cells 3076

DU 145 cells 4009

Dunning tumor 2089

DUTT1/ROBO1 3020

Dyskeratosis congenita 564, 7740

Dyskerin 7740
E-cadherin 1928, 3241, 3631, 4231, 4646, 6049, 8192

E1A 44, 329, 1028, 2534, 7991

E1A and ras oncogenes 719

E2A 6559

E2F 437, 1359, 2939, 3784, 4220, 4539, 5835, 6498, 7569

E2F-1 2376, 3715, 6278

E2F1 1563, 4414

E2F4 1563

E3-ubiquitin ligase 3677

E3L 8379

E5 248, 7808

E6 1510, 2605, 2961, 5665,8140

E6-E7 oncogenes 419

E7 oncogenes 1510

Early detection 5462

EBNA-1 5182

EBNAZA 1263

Ebp1 5609

EBV 4166, 4473, 5182

EBV transforming genes 4166

Eck 7690

ECM 8011

eed 299

EGF 119, 4071

EGF receptor 5038, 8723

EGF-R 4812

EGFP 4508

EGFR 4032, 6255

EGR-1 3391

EKLF 3368

Ela 7131

Elafin 2768

Elk-1 719, 6434

Elongation phase 3715

EMAST 4894

Embryonal carcinoma 1739, 2880

Embryonic stem cell 5504, 5515

EMMPRIN 5765

EMT 5148

End-joining fidelity 1401

Endocrine tumors 7605

Endogenous retroviruses 5593

Endometrial cancer 2815 , 3517, 7981

Endonuclease V 1909

Endoplasmic reticulum 1493

Endoplasmic reticulum stress response 8749
Endothelial cell proliferation 844

Endothelial cell(s) 2058, 3552, 4200, 7027

Endothelium 1309, 2433

Enhanced transactivation 5704

Environmental tobacco smoke 6870, 7341

Eosophageal squamous cell carcinoma 6641

Eph kinase 7690

Eph receptor tyrosine kinase 7011

Ephrin 7011

Epidemiology 7326

Epidermal growth factor 460, 2161, 4738, 6520

Epidermal growth factor receptor 2846

Epidermal keratinocyte 738

Epidermis 4266

Epigenetic modification 4822

Epigenetic(s) 3864, 4345, 5358, 5394

Epithelial cells 1590 , 2161, 7656

Epithelial proliferation 4900

Epithelium 1527

Epithelium-mesenchyme transition 2347

erbA 4307

ErbB 460, 2066, 4032, 6530

ErbB receptors 5609

ErbB1 2584

erb-B-2 8732

erb B2/neu 2805

ErbB2 78, 3934, 4567, $6255,6573,7042,7524$

erb-B-3 8732

ErbB3 8442

ErbB4 8442

ERBIN 7042

ERG 148

Erk 1/2 8871

Erk 22

ERK 307, 1978, 2309, 5923

erk 6413

ERK activation 347

Erk1/2 6255

ERK2 1370

Error-prone DNA repair 8957

ER-stress 2623

Erythroleukemia 1272 , 1859, 3562
Erythropoiesis 400, 1556 , 2641, 2864

ES oncoprotein 1695

ESET 148

Esophageal cancer 411, 475, 1208, 5024, 7745

EST1 522

Estradiol 964

Estrogen 761, 836, 1097, 5233, 7680, 7730

Estrogen-dependence 8178

Estrogen receptor $\alpha 4921$, 5233

Estrogen receptor(s) 4000, 4317, 7034, 7730, 8158

ESX 3934

Etk 8817

ETS 2309

Ets 3934

ETS transcription factors 2890

Ets-1 7933

ETV6-NTRK3 5684

ETV6/ARG 4374

EVI1 3232

Ewing family tumor(s) 3847, 8302

Ewing tumor 307

Ewing's sarcoma 809, 3847, 7700

EWS 1890

EWS-ETS 8302

EWS/FLI 3847

EWS - WT1 2009

Exchange factor 7720

Exonuclease 2130

Exonuclease activity 5117

Expression 7749

Expression analysis 1832

Expression profile 4120

Expression profiling 2948, 5566, 7256

Extinction 4908

Extracellular matrix 8506

Extracellular regulated kinase 5515

Extracellular signal regulated kinase 5601

Eye 1841

FADD 319, 4129

FAK 96, 2513, 3969, 6488, 6992

Falkor 6713

Fanconi an(a)emia 2406, 4873, 5002

Farnesylation 5998

Farnesyltransferase inhibitor (FTI) 7883 
Fas 1600, 4129, 4702, 5593

F-box 1501

F-box protein 856

Fc receptors 3677

FDXR 7195

Fer 4997

Fertility 3644

fes 3670

FGF 1841, 1978, 2433, 3889,400

FGF receptor 400, 3889

FGF signaling 8128

FGF-2 7027

FGF-3 1899

FGF-4 899

FGF8 5069

FGF-binding proteins 5733

FGF-BP 5733

FGFR4 8251

FHIT expression 2398

Fibroblast(s) 1978, 4277, 5765

Fibronectin 8037

Fibrosarcoma 8830

Fibulin-1C 1097

Fibulin-1D 1097

Fin 16184

FLIP 337

FLRG 2227

FLT3 2555

Fludarabine 4473

Fluorescence in situ hybridization 5631

Focal adhesion kinase 1450

FOG-1 3368

Follistatin 2227

Follistatin and antiproliferative 4738

Forkhead transcription factors 1556

fos 738

Fra-1 4843

Fra-2 4843

FRA3B 8713

FRA6F 7266

Fragile sites 7266, 7671

Frameshift mutation(s) 3038, 5081

FRET 356

Friend leukemia cells 7933

Friend virus 1272

Frizzled 6598

Functional cloning 6936

Functional genomics 5753

Fusion transcripts 419

Fv2 3562
Fyn 7205

$\gamma$-catenin 7497,7981

$\gamma$-irradiation 8521

$\gamma$-radiation 4969, 5897

G1 arrest 3112, 4569

G1 checkpoint 1571

G1 delay 140

G1 phase 1485

G1/S cyclins 8158

G1/S transition 5665

G2 arrest 6249

G2-M arrest 8696

G2/M transition 1233

$\mathrm{G}_{2} 8089$

$\mathrm{G}_{2}$ arrest 2102

$\mathrm{G}_{2} / \mathrm{M}$ arrest 8414

GADD45 8696

GADD45 3391, 6222

Gadd45a 6228

GADD153 2171, 4289

Gain of function 4595

GAL4 transactivation assay 2181

Galectin-3 8001

Gamma interferon 4997

Ganciclovir 2141

Gap junctions 1989, 4992

Gas 6329

Gastric cancer 2919, 4080, 6113, 6549, 8351

Gastrin 7656

GATA-1 3368

GATA1 6669

GCIP 3784

G-CSF 5346

GDNF 1739, 5582

Geminin 6624

Gene amplification 825, 5823

Gene chip 8749

Gene conversion 5929

Gene expression 929, 1423, 2433, 2634, 2741, 2768, 3121, 3253, 3814, 4403, 5380, 5814, 6366, 6510,8206

Gene expression and regulation 4879

Gene expression profile 6549

Gene expression profiling 3095

Gene expression regulation 8506

Gene filtering 4855

Gene mapping 6858

Gene promoter 5069

Gene rearrangement 1922, 5108
Gene regulation 738 , 1411, 2854, 6264, 7488

Gene repression 447

Gene silencing 3864

Gene structure 5255, 7786

Gene targeting 1841, 5504, 5906

Gene therapy 708, 1208, 4558, 5716, 6278, 6915, 8020

Genechip 1934

Genes 2270

Genetic epidemiology 7307

Genetic instability 494, 1768, 2051, 2320, 7507, 8994

Genetic susceptibilities 6870

Genetic techniques 7164

Genetic variants 5960

Genetically engineered mice 7453

Genetics 1411, 1928, 7164, 7412, 8192

Genome instability 532, 6657

Genome scan 3909

Genome stability 592, 7230

Genomic imbalances 5097

Genomic instability 503, 890, 909, 954, 2981, 6241, 6614, 7235

Genomic scanning 5414

Genomic stability 2130, 8895

Genotoxin exposure 1768

Germ cell tumour 8878

Germline 3988

Gfi-1 1571

GLI 2476

GLI genes 5529

Glia 4050

Glioblastoma 87, 5897, 5956, 7824, 8404, 8723

Glioblastoma multiforme 5038

Glioma(s) 239, 656, 921, 1325, 4212, 5601, 7164, 8011,8705

Global genomic repair 8949

Glucose 282

Glucose deprivation 337 , 6082

Glut-1 282

Glutamate transporter GLT-1 8293
Glutamine synthetase 8293

Glutathione 2762

Golgi apparatus 248, 7808

gp120 3592

gp130 460, 4778, 8186

GPI motif 3089

G-protein coupled receptor 1658

G-proteins 1171

Granulocyte 3377

Grb2 1800, 2037, 5224

Growth 4435, 5127, 7514

Growth arrest 7897

Growth control 1527

Growth factor dependence 2785

Growth factor(s) 2201, 5175,7464

Growth hormones 6970

Growth inhibition 2357, 5325,7850

GSE 6713

GSK-3 $\beta$ 2721, 4060

GSTP1 1048

GST pull-down 6729

Guanine nucleotide 7720

Guanine nucleotide exchange factor 2425

H19 gene 1625

HaCaT 7092

Haemopoietic 3068

Hamartin 4050

HAT 7241

HB-EGF 2584

HBp17 5733

hBUB1 4673

HBV 377

HBx 6606

HCC 6606

Hck 8075

HCT116 cells 4009

HCV NS5A 4801

HDAC 7241

HDAC1 3232

HDM2 1955

Head and neck cancer 1510, 2634, 4231, 4462

Head and neck neoplasm 5016

Head and neck squamous carcinoma(s) 8206, 6598

Heat shock 1090

Heat stress 4042

Hedgehog signalling 5529

HELAD1 6387

Helicase 6387 
Helix-helix interaction 5891

Helix-loop-helix protein 1812

Hematological malignancy 5758

Hematopoiesis 3260, $3262,3284,3295,3334$, $3359,3403,3414,3475$, 3855,4778

Hematopoietic cells 2641

Hematopoietic $\mathrm{Pbx}$ interacting protein 6766

Hematopoietic stem cell(s) 680, 3270, 3274

Hemizygous deletion 6567

Hemoglobin 8361

Hepatitis B Virus 2926

Hepatitis C Virus 2926

Hepatitis viruses 2593

Hepatoblastoma 4863

Hepatocellular carcinoma 789, 1225 , 1518, 1791, 2593, 2926, 4863, 5946, 7971, 8293

Hepatocyte 1548

Hepatocyte proliferation 1335

Hepatocyte proliferation and apoptosis 1539

HER-2/neu 3532

HER1/HER2 inhibitors 119

HER2 1159, 4289

HER2/neu 890

HER2/neu 5224

HER3 8442

HER4 8442

Heregulin(s) 78, 4289, $4567,4900,8442$

Hes 3855

Heterochromatin 512, 553

Heterodimer 1870

Heterozygous state 1401

HGF transgenic 1791

$\mathrm{HGF} / \mathrm{SF} 217,1000,2309$

HIF-1 282, 4212

$\mathrm{HIF} 1 \propto 2455$

High molecular form of FGF-2 6471

Histone acetyltransferase 2729

Histone deacetylase 1 8388

Histone deacetylase(s) 719, 5361, 6340, 6703, 7991

Histone H1 8397
Histone H2AX 8967

Histone H3 3103

Histone kinase 5313

Histone methylase 5361

Histone methylation 9033

Histone methyl trans-ferase 148

Histone/protein acetyltransferase 2253

'Hit and run' 825

HIV 7464

HIV-1 transcription 5797

hiwi 3988

HMGA proteins 3644

HMGA1 2971

HMGA2 3190

hMLH1 5696

hMLH1 1443, 5758

hMre11 1633

hMSH2 5696

hMSH2 5758

HNF1 8251

HNPCC 7585

hnRNP 580

hnRNP A1 8577

hnRNP K 7187

Hodgkin lymphoma 4908

Hodgkin's disease 3095, 8173

Hodgkin - Reed-

Sternberg cells 2493

Homeobox gene 798

Homologous recombination 1130

Homologous recombination 488, 954, 2873, 3076, 4176, 5002, 5188, 8981

Homology domain 7720

Homozygous deletion $1217,1461,5631$

Hormone-resistance 7861

HOS 856, 1501

Host cell factor 1

(HCF-1) 5245

$H O X$ gene 3659

Hoxa9 4247

HOXD3 798

Hp95 6801

hpttg proto-oncogene 8173

HPV 16 E6 7507

HPV 165, 5665, 5877, 6241,8140

HPV E6 5088

HPV E6/E7 2334

HPV16 6041

hRad9 7710

hRad17 7710

H-ras 5649

H-Ras 4448
Hras1 5643

H-ras mutant 5649

H-REV107-1 2829

hSNF5 6403

hSNF5/INI1/BAF47 5193

hTERT 128, 541, 611, 650, 665, 675, 769, $3130,3517,7121$

hTERT promoter 4757 , 7991

HTLV-1 7241

HTLV-1 provirus load 2466

HTLV-I 7230

hTR 650, 664

HtrA1 6684

Human 1135

Human brain tumors 8428

Human bronchial epithelial cells 7471

Human cancer cells 6498

Human carcinoma 2334

Human cells 7630

Human cervical cancer 6041

Human chromosome 3 6915

Human gene therapy 2670

Human herpesvirus 8 (HHV8) 1823

Human mammary epithelial cells 128 , 6328

Human papillary thyroid carcinoma 4830

Human papillomavirus (HPV) 419, 1510, 8713

Human skin 2991

Human T cell leukemia virus 6751

Human telomerase reverse transcriptase 5946

Human tumors 7266

Humans lens epithelial cells 3784

HUVEC 844

Hydrogen bond 7100

Hydroxyurea 7226

Hydroxyurea-mediated DNA synthesis inhibition 2079

Hypermethylation 2328 , 4231, 5450, 5696

Hypericum perforatum 1242

Hyperplasia 1899
Hyper-recombination 9008

Hypomethylation 5414

Hypoxia 3532, 6017, 7671

I-kappa B (I $\kappa$ B) 4490

I-SceI meganuclease 7671

$\mathrm{I} \kappa \mathrm{B} \quad 6510,8759$

$\mathrm{I} \kappa \mathrm{B}-\alpha$ transgene 4569

I $\kappa \mathrm{B}$ kinase (IKK) 4490

IAP(s) 260, 2283, 5763 , 6809

IB-MECA 4060

ICAM-1 5213

ICI-182,780 964

Id-1 8498

Id2 8302

IEX-1 3706

IEX-1 and transcription 6819

IFN- $\alpha 7700$

IFN- $\beta 7700$

Ifosfamide 7700

IGF-1 5673

IGF-1R competence factor 2785

IGF-I receptor 8240

IGF-II 8240

IGF-I receptor 1890

IGF1 receptor 5684

IGFBP-rP1/mac25 140

IgM 8388

IL-2 2466, 5182

IL-3 2641

IL-6 32, 2584, 3552, 6791, 8404

IL-6/LIF 8186

Imatinib 8584

Immediate early gene 3706

Immediate-early apoptosis 4237

Immortality 627

Immortalization 128, 494, 503, 598, 4200, 4577, 4873, 6328, 7121

Immune evasion 7808

Immunity 8668

Immunoglobulin heavy chain gene 4983

Immunoglobulins 1922

Immunohistochemistry 643, 4830, 8173

Immunotherapy 674

Imprinting 5400

Inbred mouse strains 5975

Induced apoptosis 5024

Inducible ras 3058

Inducible transgene 5504 
Infant leukaemia 4706

Inflammation 4266, 4728

Inflammatory mediators 6264

ING family 4462

ING3 4462

Inhibition of Bcr-Abl 8560

Inhibitor of apoptosis protein 5024

Inhibitor(s) 6413, 8541

INI1 6403

INI1/SNF5 3112

Initiation 7392

INK4a/ARF 1518, 3207, 4498

iNOS 8047

Inositol hexakisphosphate kinase 1882

INrf2 6829

Insertional mutagenesis 419, 7247

In situ hybridization 4830, 7545

Insulin 7131, 8240

Insulin growth factor-1 receptor 2785

Insuling signaling 3739

Int2/Fgf3 5548

Integration 419

Integrin affinity modulation 4448

Integrin(s) 96, 2513, 3889, 3969, 8605

Inter-specific consomic (ICS) 6680

Interferon 1251, 1882, 2295, 7957, 8741

Interferon regulatory factors 6751

Interleukin-2/15 receptor 2009

Interleukin-21 receptor gene 368

Interleukin-6 1346, 1391

Internal tandem duplication 2555

Intestinal epithelial cells 2908

Intronic enhancer 8251

Intronic response element 3050

Invasion $87,798,1812$, 4080, 4549, 5601, 6049, 6740,8705

Invasive ductal carcinoma 3241

Invasiveness 2365, 7824, 8178

Inverse PCR 4983
In vitro colony forming cells 3284

In vivo gene tracking 3274

In vivo mutant rates 7235

Ionising radiation 1108

Ionizing irradiation 4873

Ionizing radiation 2334, 4191, 6317, 6377, 6633

Ionizing radiations 2320

IRF $\gamma 2829$

IRF-1 2829, 3391, 7776

IRF5 2914

IRS-1 5684

Isoforms 8240

ITM2B $_{\mathrm{s}} 3181$

J-774 cells 3677

JAB1 3003

JAB1/CSN-5 3507

Jak 5346

JAK kinases 3334

JAK-STAT 4812

Jak-Stat signal transduction 8404

Jak2 tyrosine kinase 7137

JAM 7642

JB6 cells 2181

JCU 5574

JNK(s) 849, 1017, 15802387, 2441, 2573, 3939, 5038, 5335, 5923, 7533,8047

JSAP1 6488

Jun 738, 3391

JunB 7156

JunD 6434

Jurkat 3213, 5108

Juxtamembrane 2555

Juxtatropic membrane TNF like action 4257

K252a 4885

Kaposi's sarcoma associated herpes virus (KSHV) 1823

KDR 6307

Keratinocyte differentiation 738

Keratinocyte(s) 947, 1616, 2652, 2991, 4728, 5135

Kidney 5582

Kidney development 2948

Kinase activity 4191

Kinases 96, 6413, 6175, 7464, 7630

Kirsten-Ras (Ki-Ras) 4490

KIT 4351
KLF5 6567

KLK4 7114

Knock-in 3445

Knockout 347

K-ras 8732

K-Ras 3792

K-RAS 4301

$K$-selection 3043

$\mathrm{Ku}$ 532, 6377

$\mathrm{Ku}$ complex 512

$\mathrm{Ku}$ proteins 5725

L6 myoblasts 7027

lacI 7126

Lactogenesis 4900

Laminar flow 1450

Laminin receptor 7478

Large $\mathrm{T}$ antigen 5725

Laser capture microdissection 4587

Latent membrane protein-1 1263

LATS1 1233

Lck 7205

Leader protein 5574

LEF/TCF 6983

Lesion bypass polymerases 8957

Leucine zipper 3879

Leukaemia 4247

Leukaemias and lymphomas 3445

Leukemia 680, 1611, 2555, 3247, 3314, 3422, 4673, 5160, 7247, 8262, 8536

Leukemic cells 3213

Leukemogenesis 3260, 3414,8577

Li-Fraumeni 2961

$\mathrm{Li}$-Fraumeni syndrome 8949

Ligation mediated PCR 5743

Light chain 37060

LINE-1 5400, 5593

Linkage analysis 8356

Linkage disequilibrium 6858

Liposome 2662

Liver 32, 377

Liver mass homeostasis 1335

Liver metastasis 6689

Liver regeneration 1548

LMP1 4166, 8047

Lobular carcinoma 3241

Lobuloalveoli 4900

LOH 158, 1518, 2919, 5975, 7580, 8356

Long-patch BER 5912
Loss of heterozygosity 1225, 2398, 2593, 2840, 3961, 4462, 5631

Luca region 4521

Luminal epithelial 6573

Lung 5814, 7749, 7831

Lung adenocarcinoma 3804

Lung cancer 65, 2328, 2398, 2418, 2822, 3003, 4129, 4521, 4577, 4822, 5450, 5804, 6868, 6884, 6908, 6915, 6960, 6970, 7284, 7298, 7365, 7421, 7435, 7497, 7598, 8347, 8683

Lung cancer cells 798

Lung carcinoma 2171

Lung neoplasms 6949, 7341

Lung tumor progression gene 5814

Lung tumor(s) 2750, 6382, 6858, 8732

LY294002 1263

Lymphomagenesis 299

Lymphoma(s) 680, 3314, 3898, 4673, 4978, 5108, 5182, 5280, 6559

Lymphovascular invasion 3631

Lyn 5346

LYVE-1 7945

Macrophage 3670, 7175

Mad 5557

Mad1ER 447

$M A G E$ genes 5400

MAGI 5088

MAGI-1 8140

MAGUK 5088

Malignancies 3475

Malignancy 3459

Malignant rhabdoid tumour 6403

Malignant solid tumors 1679

Malignant transformation 2484

MAM domain 3089

Mammalian cell extracts 5204

Mammalian cells 6356

Mammalian embryo-genesis 2880

Mammalian gene amplification 7671

Mammalian RAD51 4065

Mammary 1009, 6446 
Mammary adenocarcinomas 5643

Mammary cancer 4335

Mammary carcinogenesis 2797

Mammary carcinomas 1785

Mammary epithelial cells 4289, 4747

Mammary gland 198, 291, 6366

Mammary gland development 4900, 7214

MAP kinase phosphatase 849

MAP kinase(s) 849, 1171 2805, 4071, 4921, 5038, 6356, 6540, 7839

MAPKAPK-2 1580

MAPK(s) 1017, 2513, 3670, 4000, 4323, 4567, 6413, 6434, 6649, 8498

Marked box 8388

Maspin/PAI-1 chimera 4089

Mass spectrometry 4403 , 8029

Mathematical computing 7164

Mathematical modeling 7164

Matrix metalloproteinase 1187, 3949, 4728

Max 8515

MBP-1 2775

MCF-7 breast cancer cells 2212

MCF7 4626

MCF-7 cells 2365, 4323

MCF10A 1590

M checkpoint 1717

Mcl-1 1359, 1563

MCSF 3670

MDA-7 4558

MDCK cells 1658

MDM2 2476

mdm2 2961, 4715, 8223

Mdm2 1208, 1299, 2413, 2750, 4414, 7100, 8776

MDM2 2376, 2605, 4220, 4498

MDMX 867

MdmX 8776

MDR (multidrug resistance) 1295

MDR 1945

MDS1 3232

Medullary thyroid carcinoma 2376
Medulloblastoma 1461, 7580

MEF 6713

Megakaryocyte 3359

Megakaryopoiesis 6669

Meiosis 6201

Meis 14247

MEK kinase 6791

MEKK1 4384, 5923

MEKK 38089

MEKs 2236

Melanocortin 1 receptor 8037

Melanoma 1000, 1668, 3011, 3038, 3969, 6684, 7001, 8037, 8741

Melanoma differentiation associated gene-7 708

Membrane potential 8149

MEN 2479

Menin 6434

Meningioma 239

Merlin 5990

Mesangial cell 1750

Mesenchyme 2029

Mesothelial cell 4340

Mesothelioma 1141, 1434, 2896, 4340

Met 217, 4885

Metal-dependent cleavage 9022

Metalloproteinases 2089

Metaplasia 4099

Metastasis 1800, 1812, 2245, 2504, 2822, 3541, 3631, 4129, 4932, 5127

Methotrexate 8361

Methyl binding proteins 2704

Methyl-CpG binding protein(s) 4822, 5394

Methylated DNA binding domain column chromatography 3804

Methylation 1217, 1443, 2418, 3020, 3792, 4340, 5450, 6835, 7034, 7277, 7289, 7897, 8351, 8372

Methylation spreading 5388

Methylation tolerance 2873

Methylnitrosourea 8732

MGMT 5940

MGMT 8878

Mgmt 9033

MHC class I 248, 7808

MIC-1 4212

Mice 291
Microarray 798, 1359, 1934, 2880, 3814, 5504, 6549, 7256, 7598, 7749, 8196,8361

Microarray analysis 8206

Microarrays 2009

Micrococcal nuclease 1649

Microenvironment 3262

Microsatellite instability 2873, 3253, 4894, 5081, 5696, 5758, 6299, 7289, 7585

Microtubule organization center 6195

Microtubules 5619, 6766

Migration 4080, 4765 , 7656

mIMCD3 5583

Mini-exon 5649

Minimal residual disease 7817

Mismatch recognition 1909

Mismatch repair 2840, 2873, 5934, 6299

Mismatch repair gene mutation 7585

Mispairing 2130

Mitochondria 377, 1251, 1963, 3139, 3149, 4009, 4149, 4323, 4944, 6458, 7839, 8149, 8675, 8786

Mitochondrial DNA 3780

Mitochondrial membrane permeabilization 1242

Mitogen-activated protein kinases 1539

Mitosis 437, 5619, 6154, 6184, 6201, 6249

Mitosis checkpoint 4673

Mitotic kinases 8282

MKP-1 4435

Mlh1 9033

mLin-7 2545

MLL 4706

MLN51 4422

MluI cell-cycle box

(MCB) 7786

MMAC 2357

MMC 5002

MMP 2245, 4549

MMP2 5765

MMP-9 272, 5213, 5601, 8011

MMP inhibitors 2245

MMPs 8128

MMTV/v-Ha-ras mice 8486
MnSOD 3603, 3836, 3917, 4392

Molecular detection 7289

Molecular epidemiology 7376

Molecular marker 6960

Molecular mechanisms 8547, 8605

Molecular pathogenesis 6868, 8652

Monocyte 3377

Monosomy 74849

MORF 2729

Morphogenesis 2309

Morphology 5313

Mos 6425

Motility 4435, 8272

Mouse 4696, 5814, 6858

Mouse brain tumor models 7453

Mouse knockout cells 2484

Mouse lung tumors 5960

Mouse model(s) 584, 2840, 4335, 5097, 5504, 8612, 8629

Mouse nerve sheath tumor models 7453

Mouse skin cells 3949

Mouse thymic lymphomas 6680

MOZ 2729

MPF 1717

MPS1 kinase 6161

Mre11 8967

mRNA 3095

mRNA processing 5619

Msh6 7126

MSI tumors 8062

MSP 2418

MT1-MMP 5861

MT-MMP 8011

MTG16 6703

mTOR 5325

MUC1 3631

Muc4 7524

Multidrug resistance 2365,5160

Multiple mutations 1768

Multiple myeloma 1346, 1391, 5673, 6587

Multistep tumorigenesis 3043

MuLv 6559

Muscarinic receptor 2762

Muscle differentiation 4137

Mutagenesis 2320, 4065, 8166,8895

Mutagens and carcinogens 8957 
Mutant p53 4595

Mutation detection 1909

Mutation frequency 2873

Mutational hotspots 5704, 6898

Mutational spectrum 6898

Mutation(s) 1167, 1225, 1641, 2593, 2919, 3780, 4307, 4702, 4863, 4992, 5016, 5906, 5956, 7289, 7605, 7971

Mutator phenotype 7235

Myb 400

MYC 7235

myc 165

Myc 447, 1922, 3827 , 4838, 5835, 6498, 7765, 8515

MYC 8510

$M Y C N 1848$

MyD88 3391

MyD118 3391

Myeloid 1600

Myeloid differentiation 3475

Myeloid leukemia 4849

Myeloma 2584, 5289, 6848, 8769

MYND domain 5245

Myoblast 5268

MyoD 4137, 8114

Myogenesis 2901

Myogenic differentiation 4838

Myosin II regulatory light chain 5852

MYST 2729

Nasal NK/T cell lymphoma 4702

NATH 5056

Nbk 227

NBs 4191

NBS1 8967

NCI drug screen 119

$\mathrm{N}-\mathrm{CoR} / \mathrm{mSin} 3 \mathrm{~A}$ corepressor complexes 5245

Necrosis 6091

Nectin 2545, 4108, 7642

Nek2 6184

Neo-vascularization 1309

Neoplasia 4099

Neoplasm 1295

Neu 291

Neuregulin(s) 4900, 8442

Neuroblastoma 1848 , 2097, 3552, 4549, 5823, $5967,6132,8356$

Neuroendocrine 6908

Neuronal cells 6123
Neuronal stem cells 5255

Neuropathogenesis 3592

Neuropathological evaluation 7453

Neuropilin-1 427

NF1 4978

Nf2 5990

NF-kappaB 4392

NF- $\kappa$ B 260, 1062, 1759, 2066, 2484, 2493, 3603, 3898, 3917, 4601, 4969, 5763, 5797, 6510, 6819, 8759,8852

NF-Y 8862

NGF 22

NHEJ 6471

Niche 3262, 5441

Nicotine 7412

Nicotine dependence 7349

Nijmegen breakage syndrome 8967

NIMA 6184

Nitric oxide 8047

NK- $\kappa$ B 856, 1501, 3213

NLS 4422

nm23-H1 2097

nm23-H2 2097

$N$-methyl- $N$-nitrosourea 2797

NMR 2119

N-myc 2097, 3552

$\mathrm{N}-$ myc 3879

N-Myc 4549

Non homologous end joining 2525, 5654

Non-small cell lung cancer 6936, 6877

Non-coding RNA 1625

Non-coding transcripts 3029

Non-immunoglobulin gene 368

Nonmuscle cells 5852

Nonseminoma 3909

Nore1 1381, 2713

Normal cells 688

Northern hybridization 3592

Notch 3262, 3855

Novel gene 3029, 3089

NPM-ALK signaling 1038

NQO1 2191, 5301

Nrf2 2191, 6829

NRK 1777

NSAIDs 6032, 8347

NSCLC 7497

N-terminal domain 6722

Nth 1 gene 1649
NTRK1, oncogenic activation 8334

Nuclear accumulation 5002

Nuclear export 6766

Nuclear F-actin 356

Nuclear factor-kappa B (NF- $\kappa \mathrm{B}) 3727,4490$

Nuclear import 6766

Nuclear localization 3003

Nuclear receptors 2854

Nuclear signaling 8453

Nuclear staining 2679

Nuclease 9022

Nucleolar localization 2447

Nucleolus 4498, 6703

Nucleotide damage 1649

Nucleotide excisionrepair 8949

Nucleus 4691

Nucleus, endoplasmic reticulum 1123

Null mutant 3225

NUP98 3422

Nup98-HoxA9 4247

Nur77 3925

Nutrition 3663

$\mathrm{O}^{6}$-methylguanine 8886

ODC 4328

Oesophageal cancer 6395

Oestrogen receptor 5773

OGG1 8232

Okadaic acid 3103

Oligodendroglioma 3961

Oligomerization 176, 2130

Oligomycin 8149

Oligonucleotide arrays 6289

Oligonucleotide microarray 3961

Oligonucleotides 631, 638

Oncogene cooperation 1335,8629

Oncogene cooperativity 1272

Oncogene transformation 347

Oncogene(s) 119, 697, 937, 1150, 1611, 1625, 2066, 2864, 2939, 3058, 3796, 4200, 5108, 7421, 7593, 8506

Oncogenesis 4978

Oncogenic transformation 6983

Oncostatin M 460

Oral cancer 4231

Oral cavity cancer 6480
Origin of mutations 8957

Ornithine aminotransferase 8293

Ornithine decarboxylase 3620

Osmotic shock 947

Osteoblast 7156

Osteonectin 7077

Osteosarcoma 5975

Ovarian 4435

Ovarian cancer 1097

Ovarian cancer cells 1

Ovarian cancer chromosome 6q27 387

Ovarian epithelial cells 6289

Overexpression 239, 3532, 4830, 5056

Oxidative damage 5204, 7533, 8675

Oxidative DNA damage 3571, 8905

Oxidative stress 2762, 3872, 4363

Oxidized guanine base 8895

Oxidoreduction potential 2406

Oxygen free radicals 8905

Oxygen radicals 8895

Oxytocin receptor 1658

$\mathrm{P}$ protein 7957

p0071 7042

p107 4635, 7891

p107 pocket protein 7923

p120 7067

p130 7569

P13K-AKT 307, 697

p14 ${ }^{\text {Arf }} 6779$

p14 ${ }^{\mathrm{ARF}} 2750,3149,4220$,

6898, 8372

p15 $5^{\text {INK4B }} 8372$

p16 2108

p16 ${ }^{\text {INF4a }} 4231$

p16 ${ }^{\mathrm{INK} 4 \mathrm{a}} 1876,5960$, $6328,8372,2652$

p16INK4 6908

p16INK4a 1823

p16ink4a 5193

p19 ARF 4978

p19ARF 3827

p21 1285, 2102, 3207,

3611, 4414, 5912, 6234,

$7100,7226,8114$

p21-activated kinase 3939

p218 1285

p21 $1^{\text {waf1 }} 5773$

p21 ${ }^{\mathrm{CIP} 1} 1590,8067,8089$

p21 ${ }^{\text {Cip1 }} 8486$

P21 Waf1/Cip1 7745 
p21 WAF1 1571

p26Kip1 6908

P27 1739, 7765

p27 ${ }^{\text {Kip1 }} 3003,3199,4567$,

$6587,7214,8067,8486$, 7524

p300 1556, 2253, 2455

p33 ${ }^{\text {ING1 }} 1208$

p38 849, 974, 1000, 7092 ,

7156, 7533, 8089

p38 MAPK 1978, 4601, 4567

p42 MAPK 6425

p51 2455

p53 3082, 6299

p53 1, 9, 153, 165, 176,

356, 488, 532, 592, 731,

748, 761, 867, 878, 974,

981, 990, 1090, 1187,

1208, 1285, 1299, 1304,

$1316,1325,1510,1580$,

1633, 1641, 1685, 1922,

1955, 2020, 2102, 2334,

2534, 2605, 2613, 2914,

2939, 2961, 2991, 3043,

3050, 3130, 3149, 3207,

$3525,3784,3796,3827$,

3836, 3872, 4212, 4220,

4277, 4414, 4498, 4613,

4646, 4715, 4801, 4894,

$5325,5540,5935,6123$,

6222, 6234, 6249, 6366,

6614, 6633, 6722, 6819,

7050, 7100, 7195, 7226,

7435, 7478, 7488, 7569,

7776, 7831, 7901, 7971,

$8521,8696,8949$

p53 binding sequence 7569

p53-dependent apoptosis

861

p53 expression 5704

p53 gene 5704

p53 isoforms 6722

p53-mediated DNA

damage response 1108

p53 mutation 6689, 8372

p53 responsive element

1641

p53-responsive gene 6017

p53 target 1469

p53/Trp53/TP53 190

p53-/- 6228

p63 981, 2455, 7195, 8223

p70 S6 kinase 4032, 6587

p73 974, 1304, 3050,

3796, 4715, 5897, 6898,

7195,8223

p73L 2455

p8 1685

Paclitaxel 6249
PAI-1/maspin chimera 4089

PAKs 2236

PALA 7230

Pancreas 4696

Pancreatic adenocarcinoma 2114 4587

Pancreatic cancer 4149, 4301, 5923, 6059, 7883, 8251

Pancreatic intraepithelial neoplasia 4301

Papilloma 4266

Papillomavirus 248, 947 , 1695, 7808

PAPIN 7042

Paracrine 108

PARP 5877

PARP cell survival 3130

PARP-1 1108

Patched 3507

Patched-knockout mice 7580

Pathologic mechanism 7365

PAX2 1009

Paxillin 96

PBP 861

PC12 cells 6772

PCAT 2253

PCNA 1717, 7710

PCR 7289

PCR-based cDNA subtraction 2270

PD180970 8804

PDCD2 protein 5245

Pdcd4 3076

PDGF-C 3847

PDK-1 1727, 7891

PDZ 5088, 7042, 8140

Pediatric tumor 4345

pEg3 7630

Peptide aptamer 5753

Peptide growth factor 7680

Peptidyl-prolyl isomerase 2261

Periostin 6289

Permeability transition 8149

PGP9.5/UCH-L1 3003

$\mathrm{Ph}$ positive leukemias 8652

Phage-displayed peptide library 2662

Phenotype of leukemia 8652

Pheochromocytoma 479, 1117
Philadelphia chromosome 8612 , 8643, 8652

Philadelphia chromosome leukemia 7137

Phorbol esters 4957

Phosphatase(s) 2387 . 4435, 7464

Phosphatidylinositol 3-kinase 5515, 6587

Phosphatidylinositol phosphatase 2357

Phospho-specific antibodies 2236

Phosphoinositide 3-kinase 6520

Phospholipase $\mathrm{A}_{2} 5765$

Phospholipase C 6520

Phospholipase $\mathrm{C} \gamma 28521$

Phospholipase D 3651

Phosphoramidates 638

Phosphorothioatemodified DNA-binding sites 5117

Phosphorylation 1090, 1580, 1955, 2079, 3754, 3969, 3978, 4137, 4158, 4921, 6791, 7100, 8282

Phosphorylation regulation 5031

Phosphoserine Bcr 8560

Phosphotyrosine 1079

Photoreceptor cells 4635

Photosensory pigment 9043

PI 3-kinase 2721

PI 3-kinase signaling 7891

PI-3k 5868

PI3 kinase 1945

PI3'-Kinase 4020

PI3-K 1062

PI3-kinase 4508, 7027

PI3K 207, 937, 3334, 4567, 5148

PI3k 7131

Pigment 1841

Pilar tumor 5548

Pilocytic astrocytoma 5956

Pit-1 3190

Pituitary adenomas 3190

Pituitary tumours 1217

piwi 3988

PKA 778, 4020, 7872

PKB 1263

PKC 1727, 1978, 7619

PKC $\delta 1071$

PKC-delta 7533

PKR 6278, 8379, 8741
Plasma cell 6848

Plasminogen activator 8830

Platelet-derived growth factor 1870

PLC $\varepsilon 8105$

Pleckstrin homology domain 6520

Plk3 6633

Pluripotent 8320

PLZF 6669

PML 3422, 3925, 5557, 5574, 7957

PMS2 2840

PNT2 4328

Point mutation 5891

Polo 6195

Polo-like kinase(s) 3162, 6195, 8282

Poly(ADP-ribose) polymerase 2890

Polycom-group gene 299

Polycystic kidney disease 5583

Polymerase- $\beta 731$

Polymorphism(s) 769, 4176, 6870, 8192

Polypeptide chain initiation 5325

Polyploidy 2981

Post-transcriptional regulation 7545

Postoperative recurrence 789

PPAR- $\gamma 2171$

pRb 1510, 4158

pRB 5557

pRb pathway 4680

pRb2/p130 5897

Pre B-cell 3076

Prenatal developments 2704

Preneoplasia 6366, 6915, 7298

Preneoplastic lesions 2398

Prevention 5462, 7349

Primary 1135

Primary effusion lymphoma (PEL) 1823

Proapaptotic genes 4009

Profileration 1978

Progesterone 1009

Prognosis 643, 5566

Programmed cell death 1242, 3459

Progression 5441

Prohibitin 4539, 8388

Prolactin 5047

Prolactin antagonist 5047 
Proliferation 1686, 1812, 3525, 3579, 3670, 4680, $5148,5835,5951,8037$, 8605,8817

Proline-rich motif 4932

Proline-rich domain(s) 9, 2037

Promoter 921, 4663, 4715, 7478, 7786

Promoter analysis 3696

Promoter luciferase assay 1469

Promoter methylation 7585

Promotion 7392

Promyelocytic leukaemia nuclear bodies 1633

Prophase 2328

Prostase 7114

Prostate 1135, 1899, 4099, 8453

Prostate cancer $140,153$. 1017, 1048, 1759, 2089, 2679, 3082, 3727, 4328, 4739, 5069, 5609, 6835, 7114, 7861, 8498, 8749

Prostate cancer cells 5733

Prostate specific antigen 153

Prostate tumorigenesis 5733

Prostatic acid phosphatase 3696

Proteases 5213

Proteasome 3754, 5280, 5940, 6530, 6829

Proteasome inhibitor 165

Proteinase-3 5160

Protein domains 2253

Protein-DNA complex formation 2130

Protein-DNA interaction 9022

Protein expression 7114

Protein kinase 6184

Protein kinase C 3620, 6113, 6348

Protein kinase CK2 3754, 5031, 5280

Protein kinase D 2154

Protein phosphatase 3103

Protein - protein interaction 7187

Protein stability 4384

Protein structure 2253

Protein synthesis 5325

Protein-truncating mutation 6689

Protein turnover 7776

Protein tyrosine phosphatase 2573
Protein tyrosine kinase(s) 3314, 7797

Protein tyrosine phosphatase 7067

Protein - tyrosine kinase 1079

Proteolytic degradation 867

Proteomic(s) 1625, 4403, 8029

Prothymosin $\propto 5233$

Protocadherins 7861

Prox1 7945

PRP 2662

PRSS11 6684

Psoriasis 2991

ptch 4894

PTCHI tumour suppressor gene 6007

PTEN 53, 319, 1450, 2357, 5289

PTHrP 4020

PU.1 3368, 3377

PU.1 transcription 1272

Pulmonary adenoma progression 15960

Purvalanol 6413

Pyrimidine dimers 9043

Pyrrolo-pyrimidine inhibitor 8075

qmyoD 4838

Quantative PCR 2270

Quantitative PCR 7593

Quantitative real time PCR 6480

Quantitative real-time RT-PCR 8506

Quantitative trait locus 5960

Quiescence 3403

Rabies virus 7957

rac 1945

Rac 2425, 2641

Rac1 7060

RACK1 7619

$\operatorname{Rad} 504788$

Rad51 488, 1130, 4176, 4788, 5188, 8981

Rad51 foci 5002

Radiation 1882, 2376, 2398, 4032, 7100

Radiation damage 8926

Radio-sensitization 7883

Radioresistance 5998, 6471,8683

Radon 7365

Raf 937, 3651, 5268

Raf-1 6328

Ral 7557
Ral-GDS 937

RALT 6530

Rap 1 778, 8105

RAR $\alpha 3422$

Ras 22, 53, 1381, 1527, 2309, 2425, 3334, 3579, $3827,5753,5998,6425$, $7027,7545,8105,8769$ ras 937

RASSF1 2713

RASSF1A 3792, 6835

Ras association domain 2713

Ras effectors 937

ras genes 2797

Ras mutants 5148

ras oncogene 8397

ras oncogene 4577

Ras pathways 6530

Ras-transformed $\mathrm{C} 2 \mathrm{C} 12$ cells 3739

Ras-ERK 697

RASSF1 1381

RASSF1A 4231, 4340, 7277

$\mathrm{Rb} 1196,1571,3784$, 4539, 4635, 4691, 5193, 8001, 8372, 8388, 8852

RB1 (retinoblastoma 1) 1295

RBICC1 (RBI-inducibile coiled-coil 1) 1295

RB18A 861

RC-K8 8759

Reactive oxygen species 4392, 5844, 5886

Real time RT-PCR 4171

Real-time PCR 3814

reaper 6458

Rearrangement(s) 483, 6841

Receptor 3562

Receptor tyrosine kinase 1800, 1870, 2058, 2555, 6382

Receptors 460

RECK 8347

Recombinant congenic (IRCS) strains 6680

Recombinase 5504

Recombination 522, 598, 4065, 6614

RecQ 954

RecQ helicase 9008

Redox 282, 6317

Redox regulation 2191

Reduced expression 4462

Regulation 541, 688, 964

Regulatory phosphorylation 2236
Rel 2484, 8759

Rel-Nrg 8759

RelA 2484

Renal cell carcinoma 929, 3507

Reorganization of actin

filaments 5852

Repair 3571, 6614

Repetititve sequence 789

Replication blockade 7226

Replication inhibition 488, 3715

Replication licensing 6624

Replication-associated homologous recombination 4788

Replicative senescence 4403, 981

Reporter gene assay 4983

Repression 176, 541, 2613, 5540

Reprogramming leukemic cells 3284

Resistance 2295

Restriction landmark genomic scanning 789

RET 479

RET 1739, 5582

RET/PTC1 8166

Retina 1171

Retinal pigment epithelium 6101

Retinoblastoma ( $\mathrm{Rb})$ 1325, 1823, 4635, 4691, 6908, 8114

Retinoblastoma protein 761, 4481

Retinoic acid 2181, 2880, 8852

Retinoic acid receptor 7850

Retinoic acid receptor- $\beta$ 411

Retinoids 411, 1539, 3496, 7850

Retrotransposon 5593

Retroviral insertional activation 1150

Retroviral vectors 3274

Retrovirus 8262, 8643

Retrovirus vector 2670

Reverse Northern hybridization 3592

Reversible transformation 3058

rgr 5108

Rhabdoid 5193

Rhabdomyosarcoma 2901, 3029 
Rheb 6356

Rho 1590, 2425

Rho GTPases 2901, 4020

Rho proteins 2236

Rho-kinase 5852

RhoA 8470

RhoB 5998

RhoG 207

RhoGEF 4601

Ribonucleoprotein 564

ribonucleotide reductase R2 909

Riboregulator 1625

Ribozyme-targeting 5733

RING finger 7957

Ring Finger domain 5024

RING-H2 finger 3507

Risk assessment 7376

RIZ 3038

RLGS 3909, 5414

RNA 564

RNA interference 5716 , 6041

RNA polymerase I 2447

RNA polymerase III 2961, 5540

ROS 7195

Rothmund-Thomson syndrome 9008

RPE 1841

R-Ras 4448, 7557

RRE 7187

rRNA transcription 2447

$r$-selection 3043

RT-PCR 2704, 4830, 4983, 5804, 6289

Runx 1 2729, 8428

RUNX1 3247

Runx2 2729, 7156

Runx3 8351

S phase checkpoint 3715 , 6377

SAGE (Serial Analysis of Gene Expression) 836

SAGE 929, 6366

Sam68 2037, 7187, 7205

Sarcoma 2261, 4978

Satellite DNA 5400

Saturation density 3058

Schizophrenia 7266

Sck 6307

SDHD 7605

Second messenger 44

Segregation of partly melted molecules 3804

Selenium 3663

Self-renewal 4778, 2864

Semaphorin III 427
Seminoma 3909, 3988

Senescence 532, 627, 2447, 2991, 3270, 4277, 5135, 5557, 6328, 7266, 7897

SEPTIN6 4706

Serine protease 6684

Serine theronine kinases 6649

Serine15 1090

Serpin 4089

Serum 6960

SH2 4422

SH3 2037, 4422

Shc 1800, 3872, 6307

Shc family 22

Shc family proteins 5823

Shear stress 1450

SHH 6007

ShIF 844

SHIP 5289

SHIP-1 1477

Short mononucleotide repeats 8062

SHP-2 1870

Sialyl-Lewis X/A 3631

Signal tranduction 53, $119,611,1123,1423$, 2201, 2641, 3314, 3359, 3562, 4508, 5175, 5289, 6101, 6264, 6649, 7464, 7619, 7690, 8568, 8871

Signaling 400, 6348, $7524,4812,8629$

Silencing 3792, 5358, 5380, 5388

Silibinin 1759

Single cell-based analysis 7545

Single nucleotide polymorphism 7745

Single-strand annealing 5188

Sir proteins 512

Skin 1527, 3765, 4728

Skin cancer 3836,4894 , 4969

Skin carcinogenesis 53, 1501,3620

Skp2 6587

SKY 5097

Sm proteins 5619

Smad 2227, 5783, 8776

Smad3 5660

Smad 4 158, 1616, 4696, 6049

Smad7 4879

Smad signaling 4879

Small cell lung cancer 8310
Small cell lung carcinoma (SCLC) 3814, 6877

Small intestinal epithelium 7126

Small intestine 158

smgGDS 2425

Smoh 6007

Smoking 2398, 7289, 7298, 7365, 7412

Smoking cessation 7349

SN-38 3611

Snail 3241

Socioeconomic level 7341

SOCS 4812

SOCS-1 4351

Soluble Eph receptor 7011

Somatic cell 564

Somatic cell hybrids 8536

Somatic hypermutation 483

Somatic mutation 2840

Sonic hedgehog 2670, 8196

Sorbitol 2441

SOS response 8949

Sos1 7060

SOX9 7850

SP-C 7831

Sp1 738, 7786

SPARC 7077

Specificity 1563

Spectral karyotyping (SKY) 890, 4706

Spectrin 5255

Spermatogenesis 899 , 3644, 3988

Spheroids 307

Spindle assembly checkpoint 6161

Spindle pole body (SPB) 6161, 6195

Splice mutation 4171

Splicing 5649

Sputum 6960

Squamous cancer 5135

Squamous carcinoma 3688

Squamous carcinoma subtypes 8206

Squamous cell carcinoma 1187,2670

Squamous cell carcinoma of the head and neck 2846

Src 1695, 2037, 4020, 4508, 6340, 6488, 6992, 7001, 7256, 7619, 7797, 8075,8723

Src family kinases 1707 , 3677
Src kinases 3334

Src/Yes 4879

$S$. stercoralis antigen 2466

Stability 2119

Stable transfectants 5601

Stat1 2504

Stat1 2504, 4473

Stat3 217, 1038, 3082, 3552, 3949, 4997, 6791, $7001,7611,8186,8404$

Stat3 activation 2000

Stat $3 \beta 217$

Stat5 1668, 2846, 8577, 8075,8804

Stat5a 4335

STAT(s) 3334, 3579, 5346

Staurosporine 65, 4089

Ste20 3939

Stem cell factor 1556

Stem cell(s) 3284, 3988, 4778, 5441, 8310, 8320

Steroid hormones 5175

STI571 5716, 5868, 8541

Stk 3562

Stratifin 8310

Stress kinase(s) 2573, 4384

Stress response 6017

Stroma 2029, 8830

Structure-function 4448

Structure-function relationships 9022

Suberoylanilide hydroxamic acid 427

Substrate specificity 3103 , 7205

Subtractive hybridization 3814, 7598

Suicide gene therapy 2141

SUMO 7957

SUMO-1 7913

Survival 1263, 3213, 6498, 8817

Survival signals 3651

Survivin 2613

Susceptibility 6858

SV40 190, 1141, 1434, 2896, 4328, 4340

SV40 chromosomes 5929

SV40-TAg 128

SV40 T antigen 981, 4335

SWI/SNF 5193

SWI/SNF 6403

SWI/SNF complex 3112

Synovial sarcoma 4181, 5791

Syntenin 4080 
Synteny relationship 5975

Synthetic inhibitor 2089

SYT-SSX 4181, 5791

$\mathrm{t}(16 ; 21)$-positive myeloid malignancies 6703

$\mathrm{t}(2 ; 5) 1038$

$\mathrm{t}(3 ; 16)(\mathrm{q} 27 ; \mathrm{p} 11)$ trans-location 368

$\mathrm{t}(3 ; 21) 3232$

TACC proteins 5619

$\mathrm{TAF}_{\text {II }} 317100$

Tag 2896

T-ALL 483

Tamoxifen 836, 964, $2815,3517,3603,4626$

Target gene(s) 2829, 2914, 4663, 6983, 7077

Target identification 7749

Targeted therapy 6970

Tax 7241

Tax gene amplification 7230

t-BHQ 5301

T-box 3827

Tbx3 3827

TC21 1062

T-cell activation 1571

$\mathrm{T}$ cell factor 2679

T-cell lymphoma 8173

$\mathrm{T}$ cell(s) 7205, 8668

Tcf 8340

Tcf-1 6446

TCF/LEF 8453

TCF/LEF-dependent transcription 5906

TCr 2029

Tel-Jak 4351

Telomerase(s) 494, 503, 512, 522, 541, 553, 564, 580, 584, 592, 611, 619, $627,631,643,650,656$, $664,674,680,688$, 1434, 3011, 3130, 3517, 3784, 4071, 4200, 7121, $7740,7897,8020,8262$

Telomerase activity 447

Telomerase inhibitors 638

Telomerase reverse transcriptase (hTERT) 643, 656

Telomerase RNA 656

Telomere capping 553

Telomere(s) 494, 512, 532, 553, 580, 584, 592, $598,627,680,611,619$, $825,3270,6884,8262$

Terminal exon 5649

TERT 4071
Testicular cancer 8878

Testis 899,3644

Tetracycline-inducible system 3445

TFIIH 4921

TFIIIB 5540

TFPI-2 921

TGB- $\beta$ 108, 3422

TGF $\beta$ 798, 2227, 4879, 5783, 6049, 7147, 7839, 8776

TGF- $\beta 11616,7156$

TGF- $\beta$ signaling 7883

TGF- $\beta$ type II receptor (TGF- $\beta$ RII) 7883

TGF $\beta$ type III receptor 3541

Thapsigargin 2623

The patched gene 2670

Therapeutic response 8769

Therapy 7593, 8262

Thermostable ligase 1909

Thiazolidinediones 2171

Thioredoxin 1625, 2406, 6317

Thioredoxin reductase 6317

Thrombopoietin 3359

Thymidine kinase 8340

Thymidine phosphorylase 8477

Thymocyte differentiation 299

Thymus 3765

Thyroid 778

Thyroid carcinoma 5056

Thyroid cell 2971

Thyroid hormone 761, 4307

Thyroid receptor genes 4307

Thyroid tumor 8166

TIEG 5783

Tight junctions 7642

TIMP 2245

TIP49 5835

Tissue arrays 4587

Tissue microarray 2476

Tissue specific 738

Tissue-specific expression 3696

TMA 2476

$\mathrm{TNF} \alpha 929,2441,3917$, 4237, 4384, 5024

TNFR2 signaling 4257

Tobacco 7284, 7307, 7376

Tobacco control 7349

Tobacco smoke 6898

Tobacco smoke pollution 7341
Tobacco use 7326

Tolerance 6082

Topoisomerase I 6614, 7913

TPA 1071, 6657

TPE 512

T-PLL 483

Tpo receptor 6669

TRAF proteins 2493

TRAIL 260, 337, 1135, 2283, 2623, 3139, 4323, 4558, 5763, 8510

TRAIL/Apo2L 6032

Transactivation 6606, 7226

Transcript map 6395

Transcription 153, 541, $990,1411,1812,2613$, 2961, 3422, 3898, 4843, 5540, 5660, 5783, 6340, 6751, 7933, 8862

Transcription factor $\mathrm{Sp} 1$ 1485

Transcription factor(s) 1859, 3295, 3368, 3949, 4908

Transcription initiation 7786

Transcription regulation 8477

Transcription-coupled nucleotide excision repair 5743

Transcription-coupled repair 8949

Transcriptional activation 176, 1611, 5877, 7872

Transcriptional factor 4983

Transcriptional co-repressor 6703

Transcriptional regulation 921, 1309, 3199 , 3925, 7478, 7730

Transcriptional repression 3496, 5361, 5394, 5557

Transcriptional suppression 3706

Transdifferentiation 5548

Transferrin receptor 7933

Transformation 697, 1062, 1611, 1695, 1707, 1800, 2901, 2971, 4200, 4448, 4577, 5990, 6540, 7121,8568

Transformation response 3949

Transformed 5886
Transforming activity 4374

Transforming growth factor beta 8272

Transgene 6559, 8166

Transgenic 198, 899, 1841

Transgenic mice 738 , 1028, 1548, 1899, 3190, $3445,3620,5182,6382$, $7214,7831,8732$

Transgenic mouse 6657

Transgenic rats 2797

Transgenic shuttle-phage 7126

Translation 7891

Translocation 1890, 3314, 4374

Transplantation 3270

Transrepression 2181

TRAP 665

TRAP220 861

TRC8 3507

Treatment(s) 7412, 8547

Trefoil factors 4020

TRF2 532

Trichostatin A 427, 6340

Trigger 5380

Tristetraprolin 4237

Trithorax genes 4849

Trk 22

Trp53 5643

Trp53 4635

TRRAP 5835

TSC2 8470

TSP-1 7945

TSG101 4830

TSLC1 6936

TSLL1 6936

TSLL2 6936

Tuberin 4050, 8470

Tuberous sclerosis complex 8470

Tumor angiogenesis 2000, 7011

Tumor antigen 239

Tumour-associated 3879

Tumor associated antigen 674, 5006

Tumor associated proteases 4257

Tumor auto-immunity 5006

Tumor cell survival 7001

Tumor cells 2347

Tumor control 3603

Tumor emboli 3631

Tumor growth 447, 2504, 3541,8128

Tumor immunology 6091 
Tumor-infiltrating macrophages 8830

Tumor invasion 7839

Tumor marker 4932

Tumor necrosis factor 8149

Tumor progression 6684

Tumor promoter 1071, 4266

Tumor promoter PMA 3213

Tumor promotion 3620

Tumor resistance genes 6680

Tumor-specific expression 7991

Tumor stroma targeting 4257

Tumor-stromal interaction 2009

Tumor suppression 503, 1196, 1616, 6779, 7471, 8223, 8994

Tumor suppressor 708, 1233, 1325, 1381, 2130, 3112, 3796, 4089, 4788, 5929, 7421, 7497, 7991, 8351

Tumor suppressor gene(s) 1832, 2713 , 3172, 3475, 3603, 4050, 4345, 4462, 4822, 5016, 5358, 5631, 6049, 6835 $6915,6936,5427,8356$

Tumor suppressor proteins 761

Tumor suppressors 1848

Tumor targeting 4757

Tumor xenograft 6255

Tumorigenesis 217, 282, $1800,2815,3190,4549$. 6299

Tumorigenicity 3011 , 6801

Tumors 541, 2029, 5213, 7289, 8379, 8486
Tumour angiogenesis 1309

Tumour growth 1370

Tumour necrosis factor-alpha (TNF- $\alpha)$ 6071

Tumour promotion 2387

Tumour suppressor 2119 , 2829, 2939, 4498

Tumourigenesis 7121

Two-step binding model 8967

Tylosis 6395

Type II cells 6382

Tyrosine kinase 22, 3562 , 3889, 4351, 4374, 5175, $6255,6992,8541,8568$, 8584,8643

Tyrosine kinase inhibitors 4885

Tyrosine kinase receptors 1785,8334

Tyrosine kinases 8871

Tyrosine phosphatases 8871

Tyrosine phosphorylation 2347, 6488, 7524

Tyrosol phosphorylation 6992

\section{UBC3 3978}

Ubiquitin 3754, 5940, 6829

Ubiquitin ligase(s) 856 , 1501, 6209

Ubiquitin-mediated proteolysis 8515

Ubiquitination 1079 , 1707, 4363, 6209

UCN-01 1727

UCS15A 2037

Ultrastruture 8149

uPA 7027, 7824

uPA/uPAR 8128

UPAR 2513

Uracil 8935
Uracil-DNA glycosylase 8935

Urethane 8486

Urokinase plasminogen receptor 87

UV 44

UV irradiation 1580 , 2441, 4481, 5844

UV sensitive syndrome 8949

UV-C 4065

UVA 7092

UVB 2652, 2721

UVC radiation 2079

v-Jun 6540

v-erbA 2864

V-ATPase 844

v-fms 1079

v-Myb 400, 1611

V-SEA 697

V-src 1171, 2908

Vaccinia virus 8379

Vascular endothelial cell growth factor 4765

Vascular endothelial cells 2805

Vascular tumor 2433

Vascular window model 7011

Vav 7720

VDAC 4944

VDRE 3706

VEGF 427, 1791, 2000, 2058, 2455, 2805, 2896, 6307, 7730, 7945, 8817 v-Ha-RAS 6657

VHL 929, 1117, 3507

VHL 479

VHL disease 1167

VHL gene 1167

VHR 2573

Viral integration 8713

VLA-2 2864

$v$-myb oncogene 4663

VNTR 769

von Hippel-Lindau 1117
Vulval cancer 1876

WAP domain 2768

WAVE3 5967

Werner syndrome 2447, 6657, 954, 9008

Wilms' tumor 1009 , 2009, 6694

Wilms' tumour 5566, 7277

WISP3 3172

Wnt-4 2948

Wnt antagonist 878

Wnt pathway 4060, 7971

Wnt signaling 4646, 5548

Wnt/wingless signaling pathway 5861

Wolffian duct derivatives 1899

Wortmannin 5868

WRN 2447

WT1 2948

WT1 1890

WT1 splicing 5566

Xpc mice 5704

Xenograft 3162, 7700

Xenopus laevis 1304

Xenopus oocyte 6425

Xeroderma pigmentosum 8949

XK469 4530

Xmrk 1668

X-rays 7580

XRCC3 4176

Yeast 1411, 2051

Yeast functional assay 1641, 5704

Yeast two-hybrid system 3003

YY1 8862

z-VAD 6091

Zac-1 coactivation 1469

Zinc finger 4237, 5783

ZYG-1 6201 